\title{
Atomic Ionization by keV-scale Pseudoscalar Dark Matter Particles
}

\author{
V. A. Dzuba and V. V. Flambaum \\ School of Physics, University of New South Wales, Sydney 2052, Australia \\ M. Pospelov \\ Department of Physics and Astronomy, University of Victoria, \\ Victoria, British Colombia, V8P IAI, Canada and \\ Perimeter Institute for Theoretical Physics, Waterloo, Ontario, N2J 2W9, Canada
}

(Dated: April 7, 2019)

\begin{abstract}
Using the relativistic Hartree-Fock approximation, we calculate the rates of atomic ionization by absorption of pseudoscalar particles in the mass range from 10 to $\sim 50 \mathrm{keV}$. We present numerical results for atoms relevant for the direct dark matter searches (e.g. Ar, Ge, I and Xe), as well as the analytical formula which fits numerical calculations with few per cent accuracy and may be used for multi-electron atoms, molecules and condensed matter systems.

PACS numbers: 14.70.Pw,95.35.+d,32.80.Fb
\end{abstract}

\section{INTRODUCTION}

Different lines of evidence consistently point to the existence of cold dark matter, that comprises about $1 / 5$ of the current energy budget of the Universe, 5 times more than the usual matter (see, e.g. reviews [1-5]). So far, the dark matter is seen only via its gravitational effects, which offers few clues of its true identity. Stable on cosmological time scales elementary particles with weak or super-weak interactions to visible matter naturally arise in many extensions of the Standard Model (see e.g. [1, 3] ), and may comprise the bulk of dark matter. The last two decades have seen a many-fold expansion of the experimental program aimed at direct detection of energy deposited by the dark matter particles in the collision with atoms in low radiation environments. So far no positive dark matter signal has been detected, which places important constraints on many extensions of the Standard Model that predict such particles.

Heavy stable particles (WIMPs) with masses above $1 \mathrm{GeV}$ can manifest themselves by depositing some fraction of their kinetic energy in the $\mathrm{keV}$-to- $100 \mathrm{keV}$ range in the process of scattering with atoms. Many dark matter search strategies are tuned with such signature in mind. Recently, it has been pointed out that very weakly unstable dark matter bosons in the mass range below $1 \mathrm{MeV}$ (bosonic super-WIMPs) can be detected via its absorption by atomic shells [6]. The deposited energy is dominated by the dark matter rest mass, and leads to the $\gamma$-like mono-energetic energy release. Several experimental analyses exploiting such possibility have been performed [7, 8]. (Note however, that the suggestion that the annual modulation signal observed by DAMA and DAMA-Libra [9] is caused by the absorption of superWIMPs [10] does not hold because inelastic processes do not have velocity modulation [6].) Future large-scale experiments with self-shielding capabilities [11-13] will be able to improve the sensitivity to the super-WIMPs by several orders of magnitude.

So far the calculations of absorption rate have been done using simple models of atoms which ignore the relativistic and many-body effects [6]. This way one can relate the absorption cross-sections for axial, scalar and vector dark matter particles to the photoionization crosssections. It is clear however, that atomic corrections can be non-negligible, and dedicated atomic calculations of super-WIMP absorption rates by atoms are required.

In the present work we perform $a b$ initio calculations of the ionization cross section of atoms by pseudoscalar particles using the relativistic Hartree-Fock (RHF) method. For concision, we call such a pseudoscalar particle " an axion", stressing that the mass-coupling range considered in this paper does not correspond to a QCD axion. Our calculations are not linked to the photoionization and can be directly used for the analysis of the experimental data. We consider in particular the ionization of several atoms such as argon, germanium and xenon since these elements are used in the detectors (see, e.g. 14, 15]).

We have fitted the RHF results by an analytical formula. The formula works very well when axion energy is sufficiently large to excite an electron from the $1 s, 2 s$ or $2 p$ states in atomic core. We checked that the discrepancy between the Hartree-Fock and analytical results is at the level of a few per cent for axion energy between 10 and $50 \mathrm{keV}$ and nuclear charge $18<Z<60$. The formula can be used for any multi-electron atom and wide range of axion energies. The results of this work are to be used in the experimental analyses searching for dark matter using underground detectors.

\section{THEORY}

The Hamiltonian for the pseudoscalar particle $a$ interacting with electrons can be written as [6]

$$
\hat{H}_{a}=\frac{\partial_{\mu} a}{f_{a}} \bar{\psi} \gamma^{\mu} \gamma^{5} \psi
$$

where energy scale parameter $f_{a}$ parametrizes the strength of the interaction; $\psi$ is electron Dirac field. We 
do not include the direct interaction of axion with photons $a F_{\mu \nu}^{2}$ on account of strong constraints from the $\gamma$ ray backgrounds [6]. It is convenient to present the total cross section of the atom ionization by axion in a form where the part which needs numerical analysis is presented as a dimensionless factor. Therefore we write

$$
\sigma_{a}\left(\epsilon_{a}\right)=\left(\frac{\epsilon_{0}}{f_{a}}\right)^{2} \frac{c}{v} K\left(\epsilon_{a}\right) a_{0}^{2},
$$

where $\epsilon_{0}$ is an energy scale (in our calculations $\epsilon_{0}=1$ a.u. $=27.21 \mathrm{eV}$, but it can also be any other energy unit), $c$ is speed of light, $v$ is the axion velocity in the laboratory frame, $a_{0}=0.52918 \times 10^{-8} \mathrm{~cm}$ is Borh radius, $\epsilon_{a}$ is axion energy and $K\left(\epsilon_{a}\right)$ is dimensionless factor given by

$$
K\left(\epsilon_{a}\right)=\pi\left(\frac{\epsilon_{a}}{\epsilon_{0}}\right)^{2} \epsilon_{a} \sum_{c} R_{c \epsilon}^{2}\left(2 j_{c}+1\right) .
$$

Here summation goes over all electron states $c$ in the atomic core, $2 j_{c}+1$ is the occupation number of the subshell $c, j_{c}$ is the core electron angular momentum, $R_{c \epsilon}$ is the radial integral for the Hamiltonian (1) with two single-electron wave functions. Initial electron state is the state $c$ in atomic core while final electron state is the state in continuum with the energy $\epsilon=\epsilon_{a}+\epsilon_{c}$. The integral is non-zero only between states of the same total angular momentum $j$ but of the opposite parity. The integral can be written as

$$
R_{c \epsilon}=\frac{1}{\epsilon_{a}} \int\left(f_{c}(r) g_{\epsilon}(r)-g_{c}(r) f_{\epsilon}(r)\right) d r
$$

where $f$ and $g$ are upper and lower radial components of the electron wave function in the spherically-symmetric case:

$$
\psi(\mathbf{r})=\frac{1}{r}\left(\begin{array}{c}
f(r) \Omega_{j l m}(\mathbf{n}) \\
i \alpha g(r) \tilde{\Omega}_{j l m}(\mathbf{n})
\end{array}\right) .
$$

Here $\alpha$ is the fine structure constant ( $\alpha \approx 1 / 137.036$ ). The states in the continuum are normalized to the $\delta$ function of the energy. Notice that we use realistic estimates of velocities of dark matter particles, $v / c \sim 10^{-3}$, which allows to good accuracy retain only the time derivative term in Eq. (1), and the only velocity dependence is then $1 / v$ in Eq. (2).

We use relativistic Hartree-Fock (RHF) method to calculate $K$ using (3) and (4). We will focus on the axion mass window $10 \mathrm{keV}<\epsilon_{a}<50 \mathrm{keV}$, which is in the maximal sensitivity range for the dark matter search experiments. Above these energies, the axion-induced Compton like scattering ae $\rightarrow e \gamma$ may dominate over direct axio-absorption [6]. As stated before, we consider cold non-relativistic axions so that we can always assume that $\epsilon_{a}=m_{a} c^{2}$, where $m_{a}$ is axion mass.

In principle, formula (3) can be used at any axion energy. However, at low energy, when only outer atomic electrons can be excited, RHF approximation might be insufficiently accurate due to many-body and environmental effects not included into the RHF potential. On the other hand, when ionization is dominated by excitation from $K$ and $L$ atomic shells these effect can be neglected. As known, the correlation corrections to RHF results for inner electrons $1 s, 2 s, 2 p$ decrease as $1 / Z$ where $Z$ is the nuclear charge. Moreover, RHF often gives reasonable results for $1 s$ even for $Z=2$. As a next step, the cross section can be approximated by an analytical formula which fits RHF results. The advantage of having such formulae is obvious: the results can be easily obtained for any atom.

For the non-relativistic operator (but relativistic wave functions) the radial integral (44) becomes (in atomic units)

$$
R_{c \epsilon}=\int\left(f_{c}(r) f_{\epsilon}(r)+\alpha^{2} g_{c}(r) g_{\epsilon}(r)\right) r d r
$$

This is the same form of the radial integral which appear in the photoionization cross section. In this case the expressions for the ionization cross sections by axion and photon are very similar and differ by a factor only. It was found in Ref. [6] that the cross section of atom ionization by non-relativistic axions can be expressed via the photoionization cross section $\sigma_{\gamma}$,

$$
\frac{\sigma_{a} v}{\sigma_{\gamma}\left(\hbar \omega=\epsilon_{a}\right) c} \approx \frac{3 \epsilon_{a}^{2}}{4 \pi \alpha f_{a}^{2}} .
$$

Using expressions (2) and (7) we can present photoionization cross section in a form

$$
\sigma\left(\epsilon_{\gamma}\right)=\frac{4}{3} \pi \alpha\left(\frac{\epsilon_{0}}{\epsilon_{\gamma}}\right)^{2} K\left(\epsilon_{\gamma}\right) a_{0}^{2}
$$

which uses the same dimensionless function of energy $K(\epsilon)$ (3) as the axion cross section. This gives us an opportunity to check numerical calculations using experimental data for the photoionization. We have done this for the cases of krypton and xenon using the data from Ref. [16]. We found that numerical and experimental results agree within a few per cent accuracy with an exception of the near the threshold ionization where the difference is a little higher than $10 \%$.

Expression (77) helps us to find an analytical formula for the atomic ionization by axion absorption. Analytical expressions for photoionization in Coulomb field can be found in many quantum mechanics textbooks (see, e.g. [17, 18]). Using the textbook expressions for photoionization from $1 s, 2 s$ and $2 p$ Coulomb states and the expression (7) we firstly obtain the results for a single electron atom. Then we use additional parameters to fit the numerical results of the relativistic Hartree-Fock calculations. This way we arrive to the formulae which describe ionization of multi-electron atoms by absorption of axion:

$$
K_{\text {total }}=K_{1 s}+K_{2 s}+K_{2 p},
$$


TABLE I: Hartree-Fock energies of the core states of Na, Ar, Ge, I and Xe (atomic units, 1 a.u. $=27.21 \mathrm{eV}$ ).

\begin{tabular}{|c|c|c|c|c|c|}
\hline Atom & $\mathrm{Na}$ & $\mathrm{Ar}$ & $\mathrm{Ge}$ & I & $\mathrm{Xe}$ \\
\hline$Z$ & 11 & 18 & 32 & 53 & 54 \\
\hline $1 s_{1 / 2}$ & -40.54 & -119.1 & -411.0 & -1225. & -1277. \\
\hline $2 s_{1 / 2}$ & -2.805 & -12.41 & -53.45 & -193.0 & -202.4 \\
\hline $2 p_{1 / 2}$ & -1.522 & -9.631 & -47.33 & -180.5 & -189.6 \\
\hline $2 p_{3 / 2}$ & -1.514 & -9.547 & -46.14 & -169.5 & -177.7 \\
\hline $3 s_{1 / 2}$ & -0.1823 & -1.286 & -7.409 & -40.52 & -43.01 \\
\hline $3 p_{1 / 2}$ & & -0.5953 & -5.324 & -35.34 & -37.66 \\
\hline $3 p_{3 / 2}$ & & -0.5878 & -5.157 & -33.21 & -35.32 \\
\hline $3 d_{3 / 2}$ & & & -1.616 & -24.19 & -26.02 \\
\hline $3 d_{5 / 2}$ & & & -1.591 & -23.75 & -25.53 \\
\hline $4 s_{1 / 2}$ & & & -0.5687 & -7.759 & -8.430 \\
\hline $4 p_{1 / 2}$ & & & -0.2821 & -5.868 & -6.452 \\
\hline $4 p_{3 / 2}$ & & & -0.2730 & -5.450 & -5.982 \\
\hline $4 d_{3 / 2}$ & & & & -2.341 & -2.711 \\
\hline $4 d_{5 / 2}$ & & & & -2.274 & -2.633 \\
\hline $5 s_{1 / 2}$ & & & & -0.8762 & -1.010 \\
\hline $5 p_{1 / 2}$ & & & & -0.4341 & -0.4925 \\
\hline $5 p_{3 / 2}$ & & & & -0.3903 & -0.4398 \\
\hline
\end{tabular}

$$
\begin{aligned}
K_{1 s}= & f_{1}\left(Z, \epsilon_{a}+\epsilon_{1 s}\right) \frac{384 \pi \epsilon_{1 s}^{4}}{\left(\epsilon_{0} Z \epsilon_{a}\right)^{2}} \frac{e^{-4 \nu_{1} \operatorname{arccot} \nu_{1}}}{1-e^{-2 \pi \nu_{1}}} \\
K_{2 s}= & f_{2}\left(Z, \epsilon_{a}+\epsilon_{2 s}\right) \frac{6144 \pi e_{2}^{3}}{\epsilon_{0} \epsilon_{a}^{2}}\left(1+3 \frac{e_{2}}{\epsilon_{a}}\right) \\
& \times \frac{e^{-4 \nu_{2} \operatorname{arccot}\left(\nu_{2} / 2\right)}}{1-e^{-2 \pi \nu_{2}}} \\
K_{2 p}= & f_{2}\left(Z, \epsilon_{a}+\epsilon_{2 p}\right) \frac{12288 \pi e_{3}^{4}}{\epsilon_{0} \epsilon_{a}^{3}}\left(3+8 \frac{e_{3}}{\epsilon_{a}}\right) \\
& \times \frac{e^{-4 \nu_{3} \operatorname{arccot}\left(\nu_{3} / 2\right)}}{1-e^{-2 \pi \nu_{3}}},
\end{aligned}
$$

where $\alpha$ is the fine structure constant, $Z$ is nuclear charge, $\epsilon_{a}$ is axion energy, $e_{2}=\left|\epsilon_{2 s}\right|, e_{3}=\left|\epsilon_{2 p}\right|$, $\nu_{1}=\sqrt{-\epsilon_{1 s} /\left(\epsilon_{1 s}+\epsilon_{a}\right)}, \nu_{2}=2 \sqrt{-\epsilon_{2 s} /\left(\epsilon_{2 s}+\epsilon_{a}\right)}, \nu_{3}=$ $2 \sqrt{-\epsilon_{2 p} /\left(\epsilon_{2 p}+\epsilon_{a}\right)}$. Here $\epsilon_{1 s}, \epsilon_{2 s}$ and $\epsilon_{2 p}$ are the HartreeFock energies of the core states. Hartree-Fock energies of all core states of $\mathrm{Na}, \mathrm{Ar}, \mathrm{Ge}, \mathrm{I}$ and Xe are presented in Table I. For other atoms extrapolation formulas can be used:

$$
\begin{aligned}
\frac{\epsilon_{1 s}}{\epsilon_{0}}(Z)= & -\frac{Z^{2}-7.49 Z+43.39}{2}, \\
\frac{\epsilon_{2 s}}{\epsilon_{0}}(Z)= & -0.000753 Z^{3}-0.028306 Z^{2} \\
& -0.066954 Z+2.359052, \\
\frac{\epsilon_{2 p}}{\epsilon_{0}}(Z)= & -0.000739 Z^{3}-0.027996 Z^{2} \\
& +0.128526 Z+1.435129 .
\end{aligned}
$$

The functions $f_{1}(Z)$ and $f_{2}(Z)$ in (101112) are scaling functions:

$$
\begin{aligned}
f_{1}(Z, \epsilon)= & \left(5.368 \times 10^{-7} Z-1.17 \times 10^{-4}\right) \epsilon / \epsilon_{0} \\
& -0.012 Z+1.598
\end{aligned}
$$

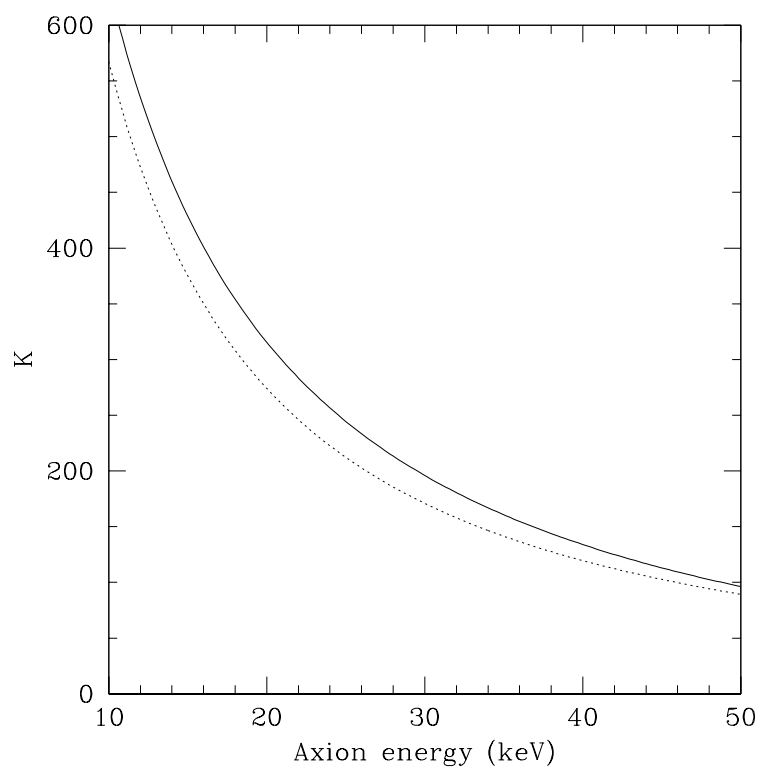

FIG. 1: Dimensionless factor $K$ (see formula (3) in the ionization cross sections of Ar by axion. Solid line - result of Hartree-Fock calculations, dotted line - formula (9).

$$
\begin{aligned}
f_{2}(Z, \epsilon)= & \left(-1.33 \times 10^{-6} Z+1.17 \times 10^{-4}\right) \epsilon / \epsilon_{0} \\
& -0.0156 Z+1.15
\end{aligned}
$$

They are chosen to fit the results of Hartree-Fock calculations for axion energies between 10 and $50 \mathrm{keV}$ and nuclear charge $18<Z<60$. We stress once more that not only scaling functions but all the formulae (10, 17) were obtained by fitting the Hartree-Fock calculations. All atomic shells are included into the RHF calculations. Therefore, the fit with $K_{\text {total }}=K_{1 s}+K_{2 s}+K_{2 p}$ includes the higher shells as well. As it will be demonstrated in the next section, the analytical and Hartree-Fock results agree within few per cent.

\section{RESULTS}

Figures 123] show the results of relativistic HartreeFock and analytical calculations of the dimensionless factor $K$ which stands in the expression for the cross section of atom ionization by axion (see formula (2)). We use the RHF computer code and formulae (3) and (4) for the calculations. Many body and relativistic effects beyond the RHF method are ignored and the final electron state in the continuum is calculated in the same potential as initial core state. The accuracy of this approximation is few percents due to dominating contribution from the inner-most core states $1 s, 2 s$ and $2 p$. For these states the many-body effects are small due to strong nuclear field.

Calculations using analytical formula (910]1112) are shown on Figures [12/3 as dotted lines. The agreement between RHF and analytical results is very good for all 


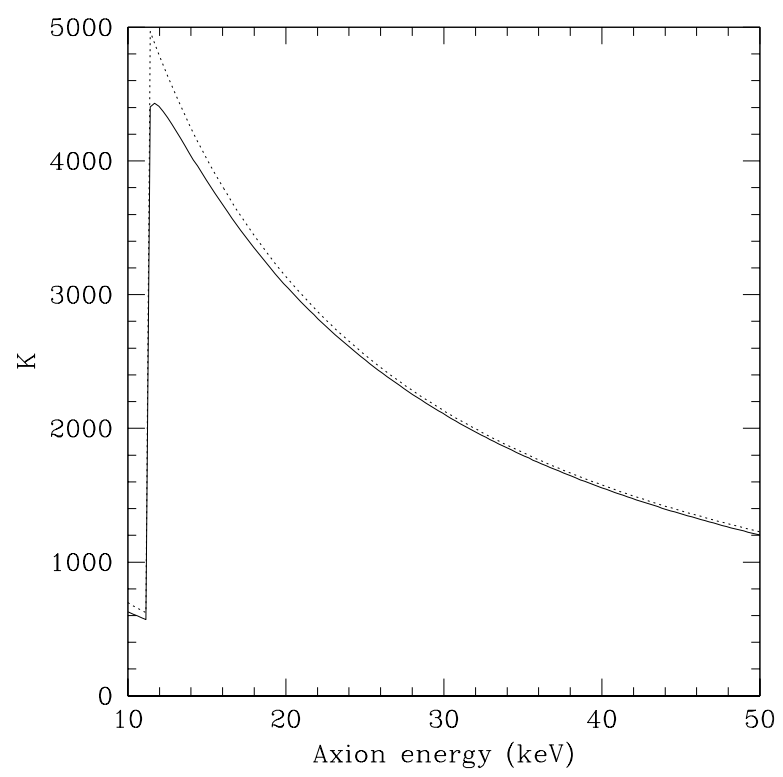

FIG. 2: As on Fig. 1 but for Ge.

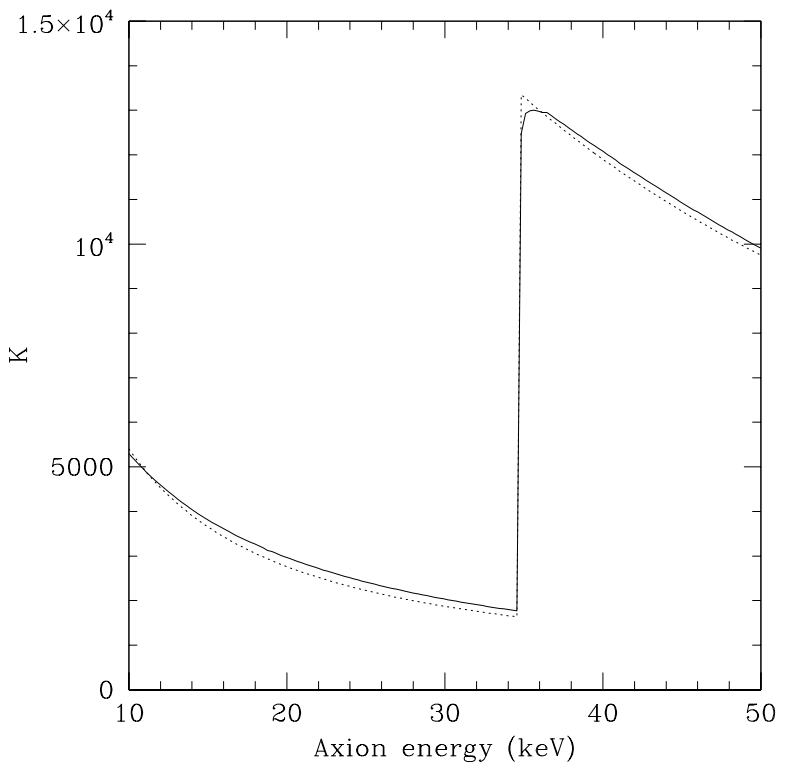

FIG. 3: As on Fig. 1 but for Xe. three atoms. It is on the order of a few per cent when an electron from the $1 s$ is excited and better than $20 \%$ when only $2 s$ and higher states contribute to the cross section. We expect similar trend for other atoms. This means that at present stage the analytical formulae (9]10]11]12) are sufficiently accurate and there is no need for more sophisticated atomic calculations.

The results show an obvious advantage of using heavy elements in the detectors given that axion energy is sufficiently large to excite an electron from an inner-most subshell. For example, for a solar axion of $\epsilon_{a}=14.4 \mathrm{keV}$ [19], $K=450$ for the case of $\mathrm{Ar}$ and $K \approx 4000$ for the case of Ge and Xe. Current experimental sensitivity to the $f_{a}$ parameter is in the range of $10^{8}-10^{9} \mathrm{GeV}$ [7, 8], but is poised to be improved in the very near future.

In this paper we have examined the case of atomic ionization by the pseudoscalar super-weakly interacting dark matter, but a similar case can be made for other types of super-WIMPs with arbitrary integer spin, and coupled to electrons via vector, axial-vector, tensor etc couplings. Another obvious use of the RHF calculations relevant to the underground searches of exotic particles, is the generalization to the detection of nearly massless particles emitted by the solar interrior with typical energies of a few keV. Both applications will be considered in a forthcoming publication.

\section{Acknowledgments}

The authors are grateful to M. Kuchiev and J. Berengut for useful discussions. The work was supported in part by the Australian Research Council.
[1] G. Jungman, M. Kamionkowski, and K. Griest, Phys. Rep. 267, 195 (1996).

[2] G. Bertone, D. Hooper, and J. Silk, Phys. Rep. 405, 279 (2005).

[3] F. D. Steffen, Europ. Phys. J. C 59, 557 (2009).

[4] E. Komatsu, J. Dunkley, M. R. Nolta, et al, Astrophys. J. Supplement Series 180, 330 (2009).

[5] G. Carosi, K. van Bibber, and M. Pivovaro, Contemporary Physics 49, 281 (2008).

[6] M. Pospelov, A. Ritz, M. Voloshin, Phys. Rev. D 78,
115012 (2008).

[7] C. E. Aalseth et al. [CoGeNT Collaboration], Phys. Rev. Lett. 101, 251301 (2008) [Erratum-ibid. 102, 109903 (2009)] arXiv:0807.0879 [astro-ph]].

[8] Z. Ahmed et al. [CDMS Collaboration], Phys. Rev. Lett. 103, 141802 (2009) arXiv:0902.4693 [hep-ex]].

[9] R. Bernabei et al. [DAMA Collaboration], Eur. Phys. J. C 56, 333 (2008) arXiv:0804.2741 [astro-ph]].

[10] R. Bernabei et al., Int. J. Mod. Phys. A 21, 1445 (2006) arXiv:astro-ph/0511262. 
[11] E. Aprile, AIP Conf. Proc. 1115, 355 (2009).

[12] S. Fiorucci et al., arXiv:0912.0482 [astro-ph.CO].

[13] A. Hime, AIP Conf. Proc. 870, 205 (2006).

[14] F. T. Avignone, Phys. Rev. D 79, 035015 (2009).

[15] S. Andriamonje, S. Aune, T. Dafni, et al, Nuc. Instr. Meth. Phys. Research A 535, 309 (2004).

[16] F. Wuilleumier, Phys. Rv. A 6, 2067 (1972).

[17] V.B. Berestetskii, E.M. Lifshitz, and L.P. Pitaevskii,
Quantum electrodynamics, Butterworth-Heinemann Ltd (1982).

[18] H. A. Bethe and E. E. Salpeter, Quantum mechanics of one- and two-electron atoms, Spinger-Verlag (1957).

[19] S. Andriamonje, S. Aune, D. Autiero, et al, J. Cos. Astr. Phys. 12, 002 (2009). 